\title{
Searching for Ultra-Faint Milky Way Satellites with the DELVE Survey
}

William Cerny (on behalf of the DELVE collaboration)

New Perspectives 2020

$7 / 21 / 2020$

This document was prepared by DELVE collaboration using the resources of the Fermi National Accelerator Laboratory (Fermilab), a U.S. Department of Energy, Office of Science, HEP User Facility. Fermilab is managed by Fermi Research Alliance, LLC (FRA), acting under Contract No. DE-AC02-07CH11359.

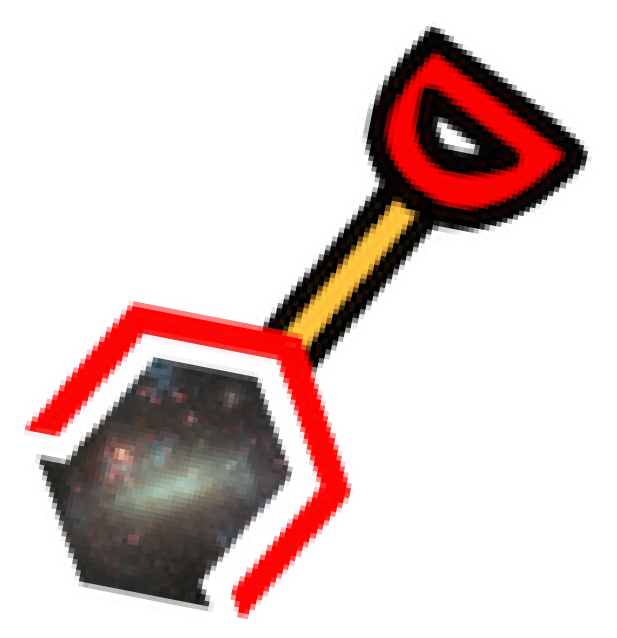




\section{The Universe's Least Luminous Galaxies}

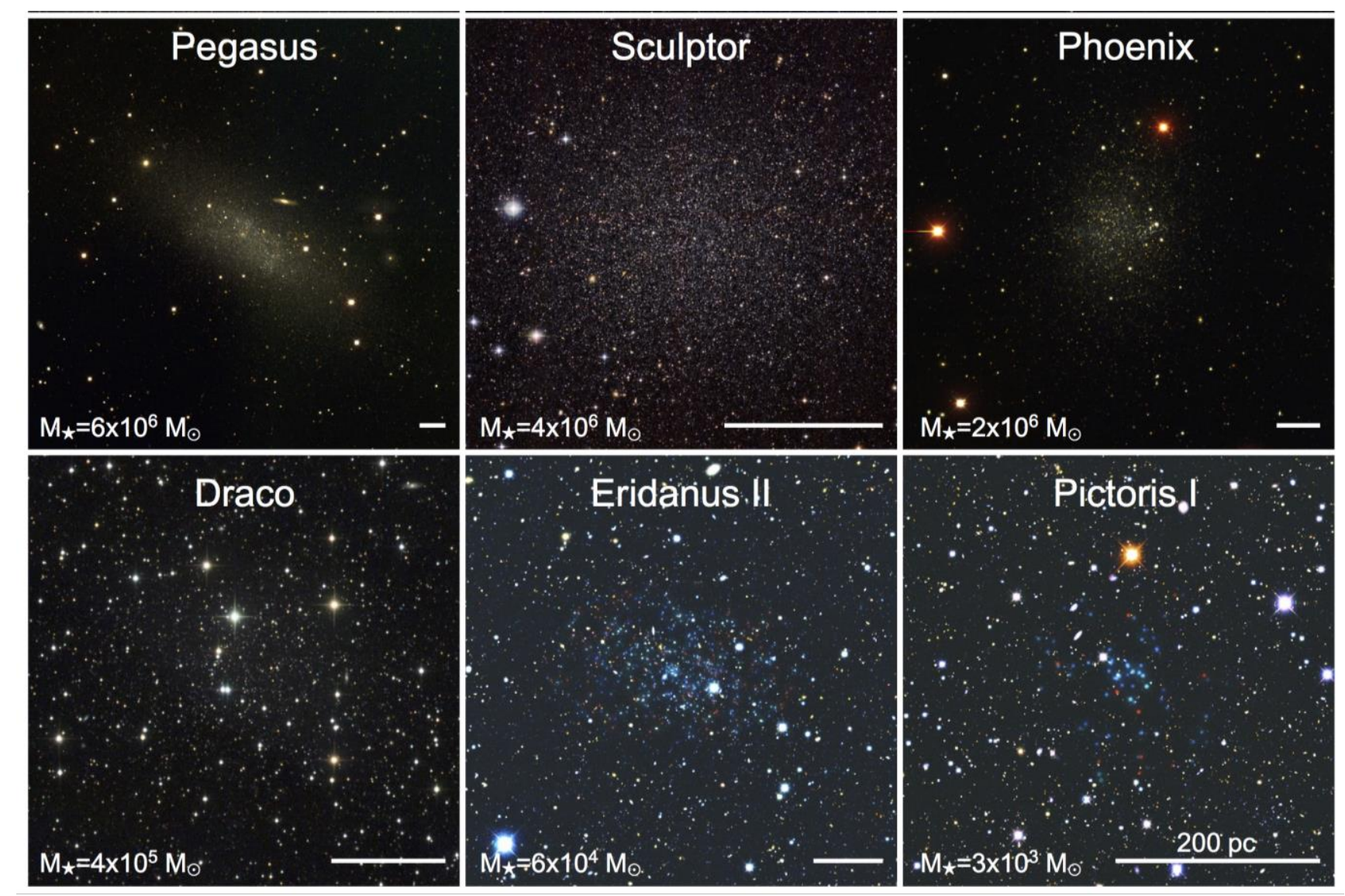

Adapted from Bullock \& Boylan-Kolchin (2017)
- Diffuse and Low Surface Brightness -> hard to detect!

- Less compact and much fainter than globular star clusters

- Only 60 known Milky Way satellite dwarf galaxies

- Old, metal poor stellar populations

- Often only tens to hundreds of detectable member stars

- Dark Matter Dominated: 99\% dark matter

- Inhabit substructure in MW halo

- Masses typically up to $100 x$ bigger than what would be measured from luminous matter alone 


\section{Why Study Dwarf Galaxies?}

\section{Test Dark Matter Particle Models}

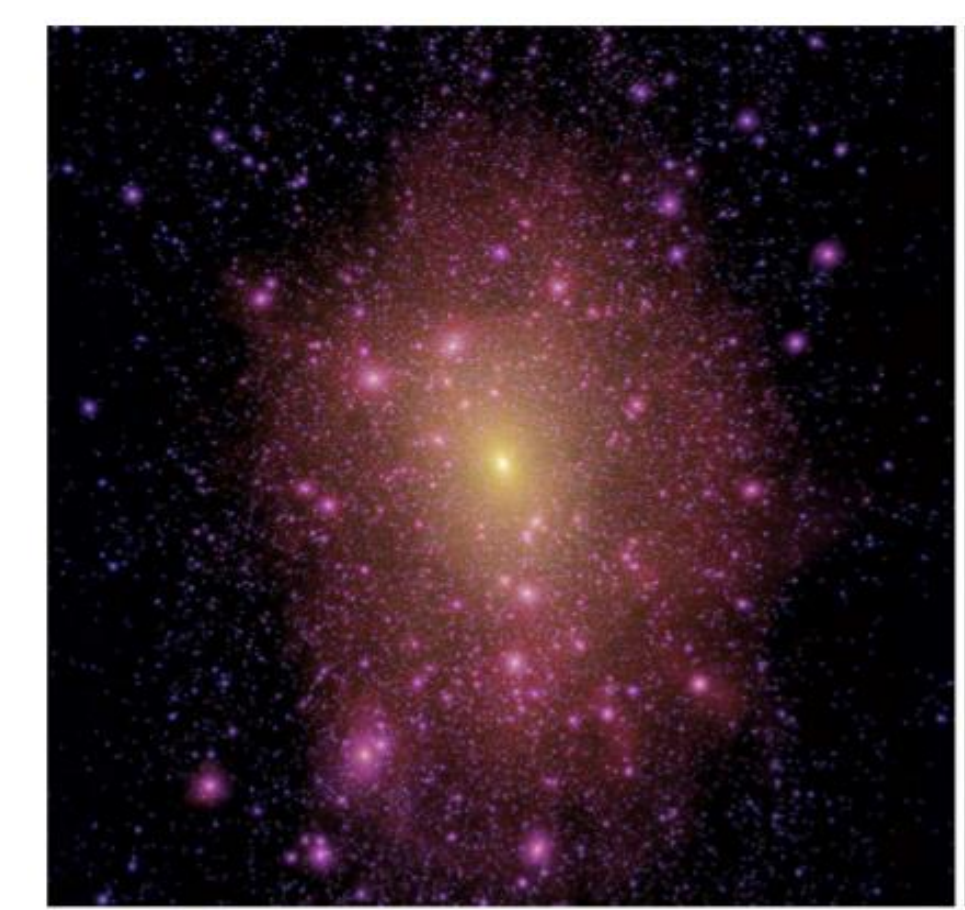

Cold Dark Matter Simulation

Image credit: Lovell et al. (2012)

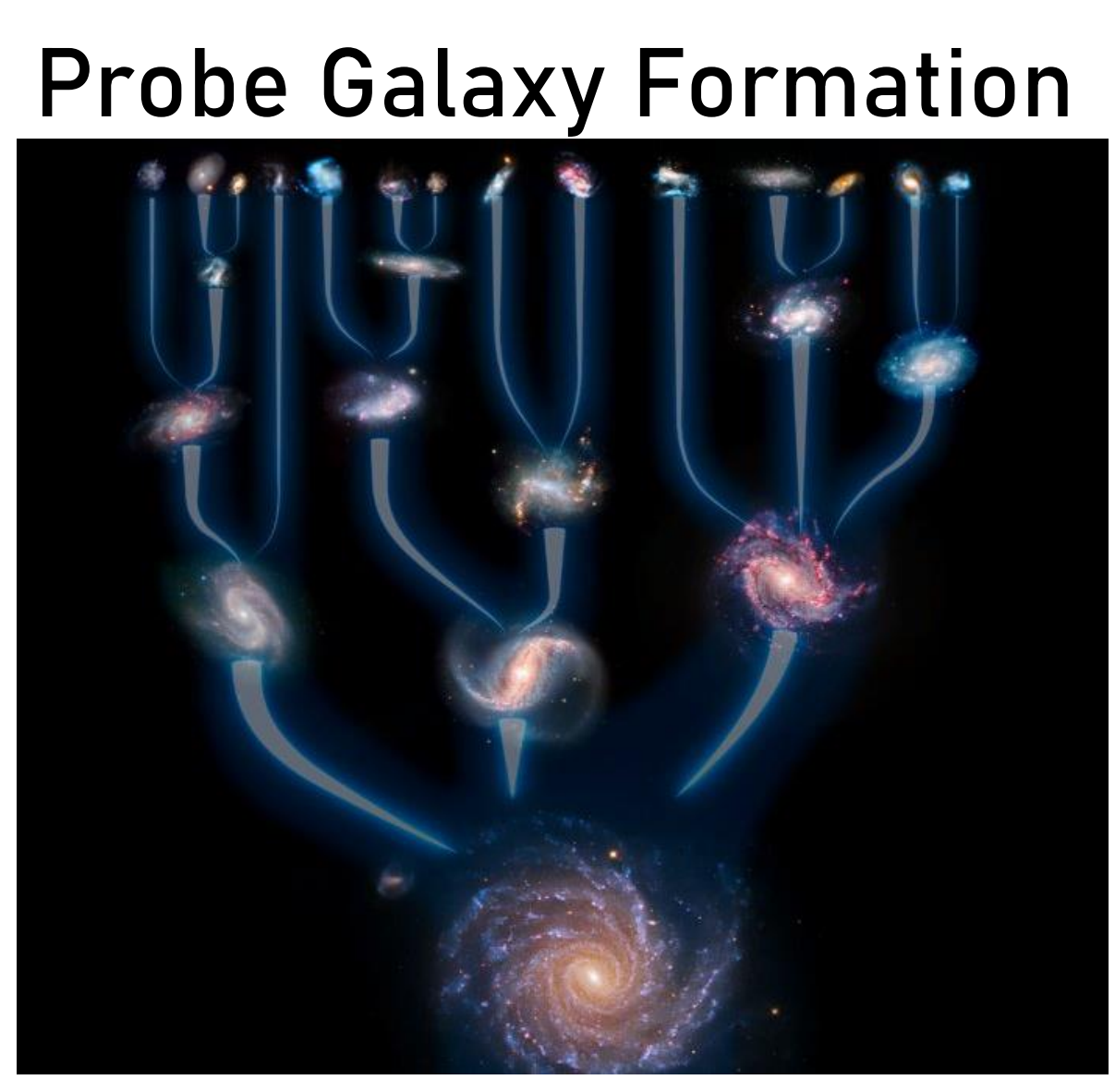

Image credit: ESO 


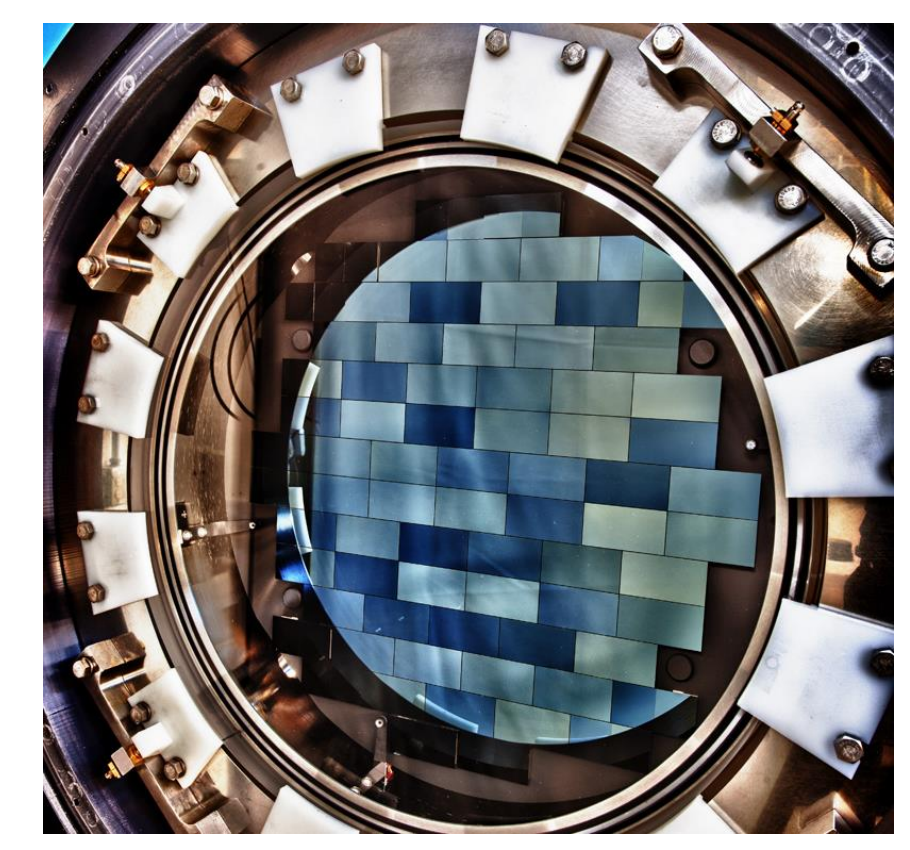

Image credits: Dark Energy Survey Collaboration

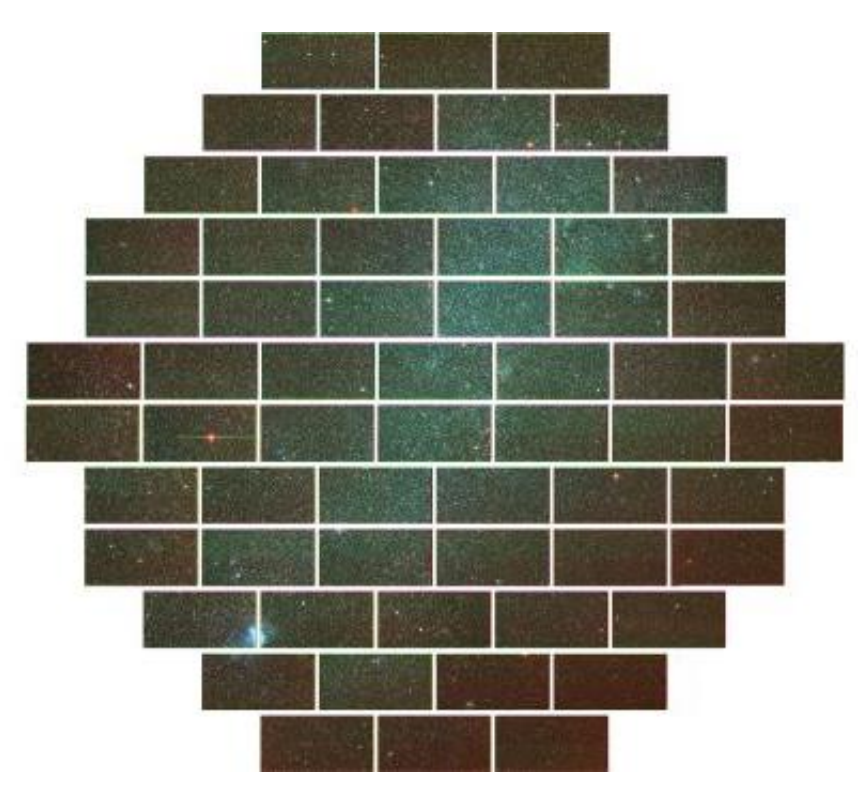

\section{The Dark Energy Camera}

- Originally built for the Dark Energy Survey Collaboration, centered here at Fermilab

- Mounted on 4-meter Blanco Telescope at Cerro Tololo Inter-American Observatory (CTIO) in Chile

- Extremely-well suited for wide-field sky surveys:

- Large field of view ( 3.4 deg^2)

- High Resolution: 62 4,000×2000 pixel CCDs - 570 Megapixel camera!

- Used to discover 20+ dwarf galaxies to date 


\section{Milky Way Satellite Discovery Timeline}

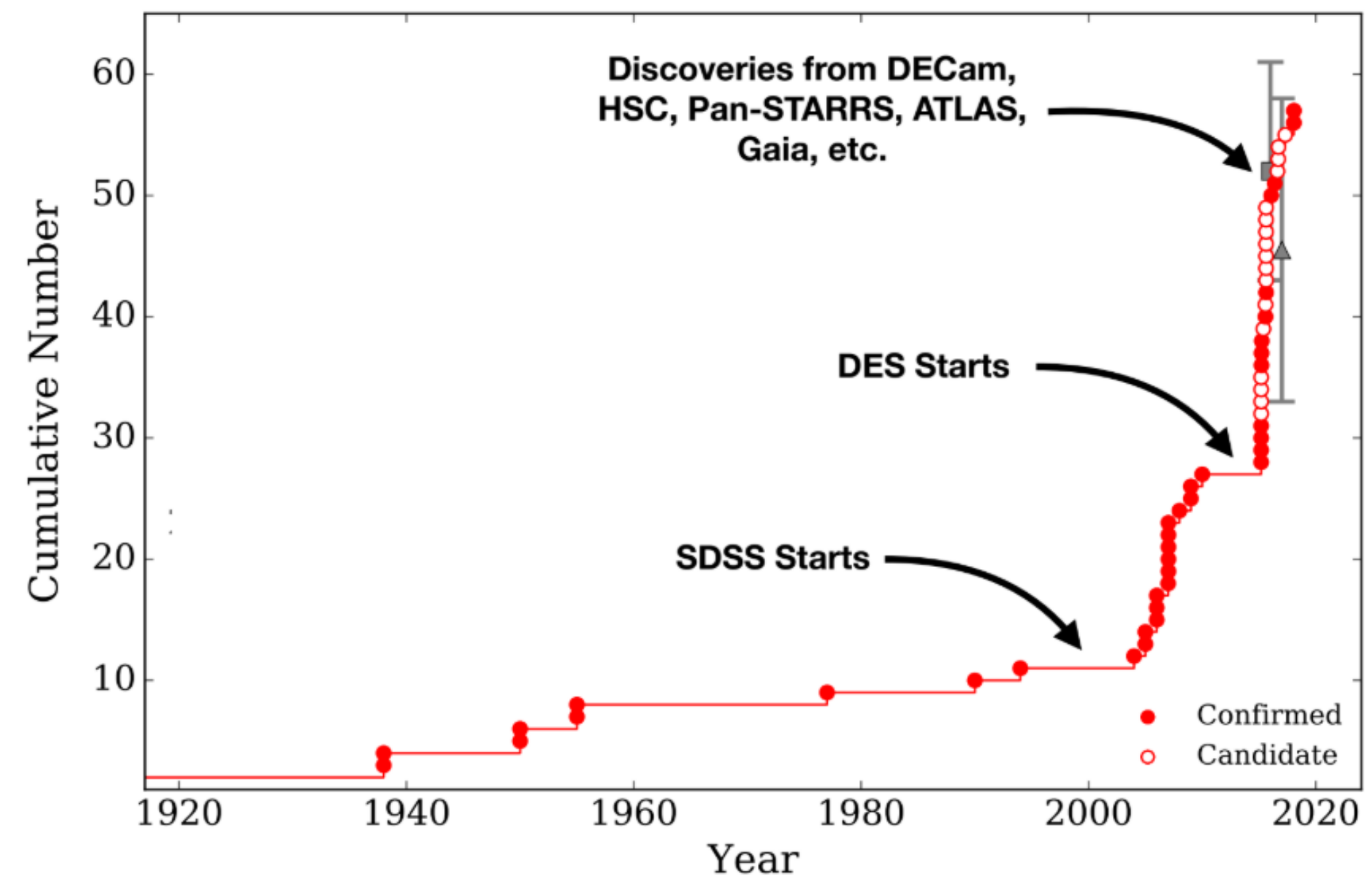

Image credit: Alex Drlica-Wagner 


\section{DECam Local Volume Exploration (DELVE)}

- Aiming to complete uniform, contiguous coverage of the Southern Sky on the Dark Energy Camera (DECam)

- Combines 126 nights of new g- and i- band observations across 3 years with thousands of publicly available exposures taken on the same instrument from the community

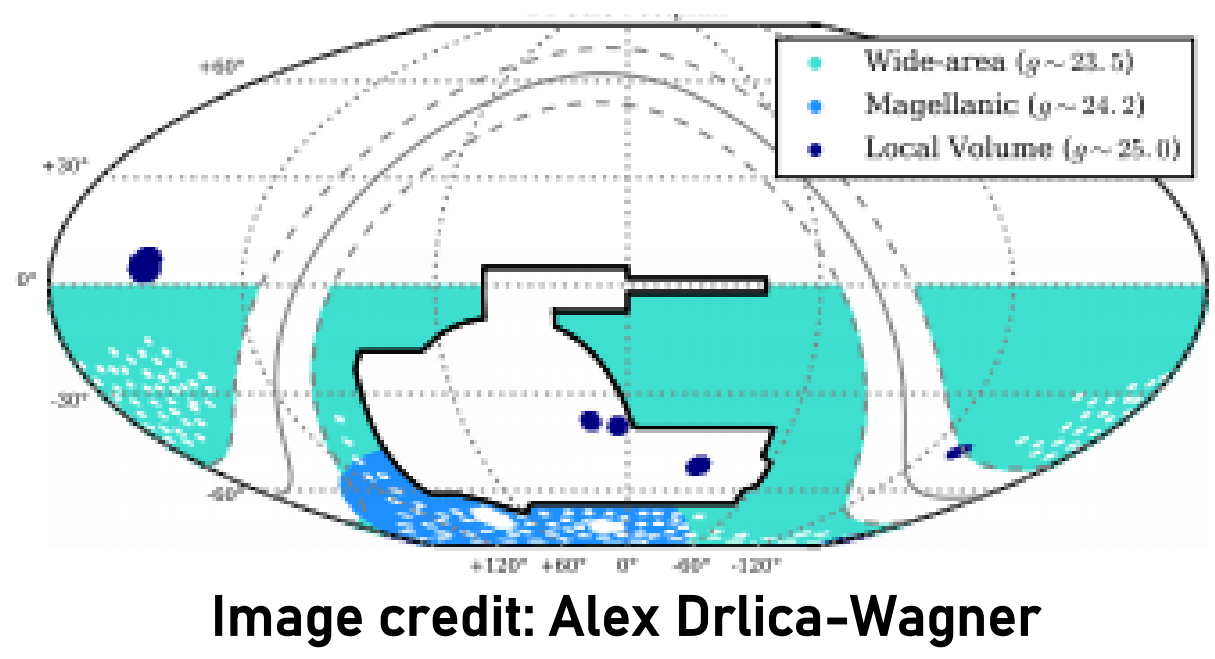

- Perfect for conducting a census of Milky Way dwarf galaxies! (among many other science cases)

DELVE Collaboration:

85 members across 10 countries! 


\section{Searching for Dwarf Galaxies}
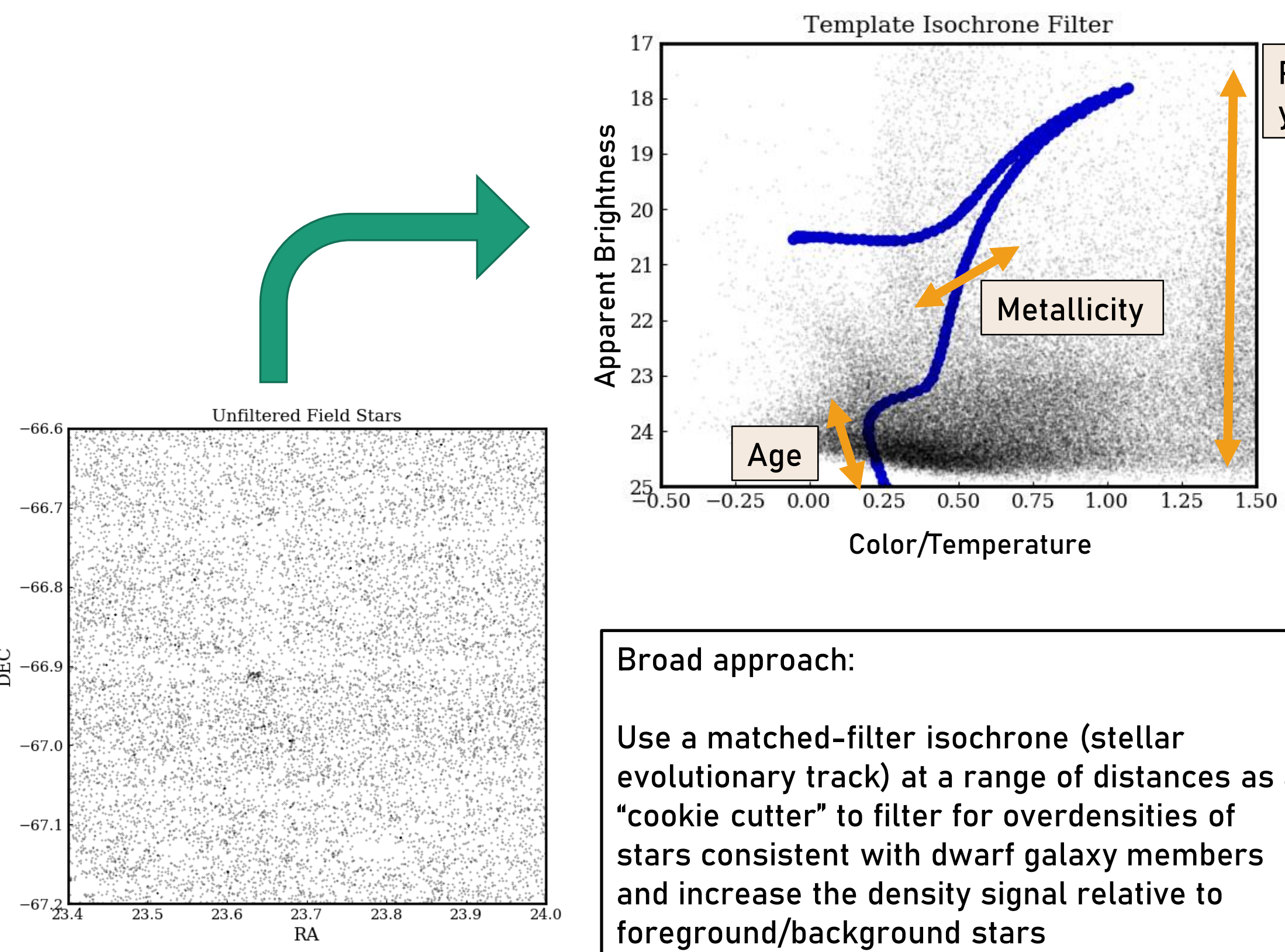

Position of entire isochrone on

$y$-axis is fixed by Distance

\section{Broad approach:}

Use a matched-filter isochrone (stellar evolutionary track) at a range of distances as a "cookie cutter" to filter for overdensities of stars consistent with dwarf galaxy members and increase the density signal relative to foreground/background stars

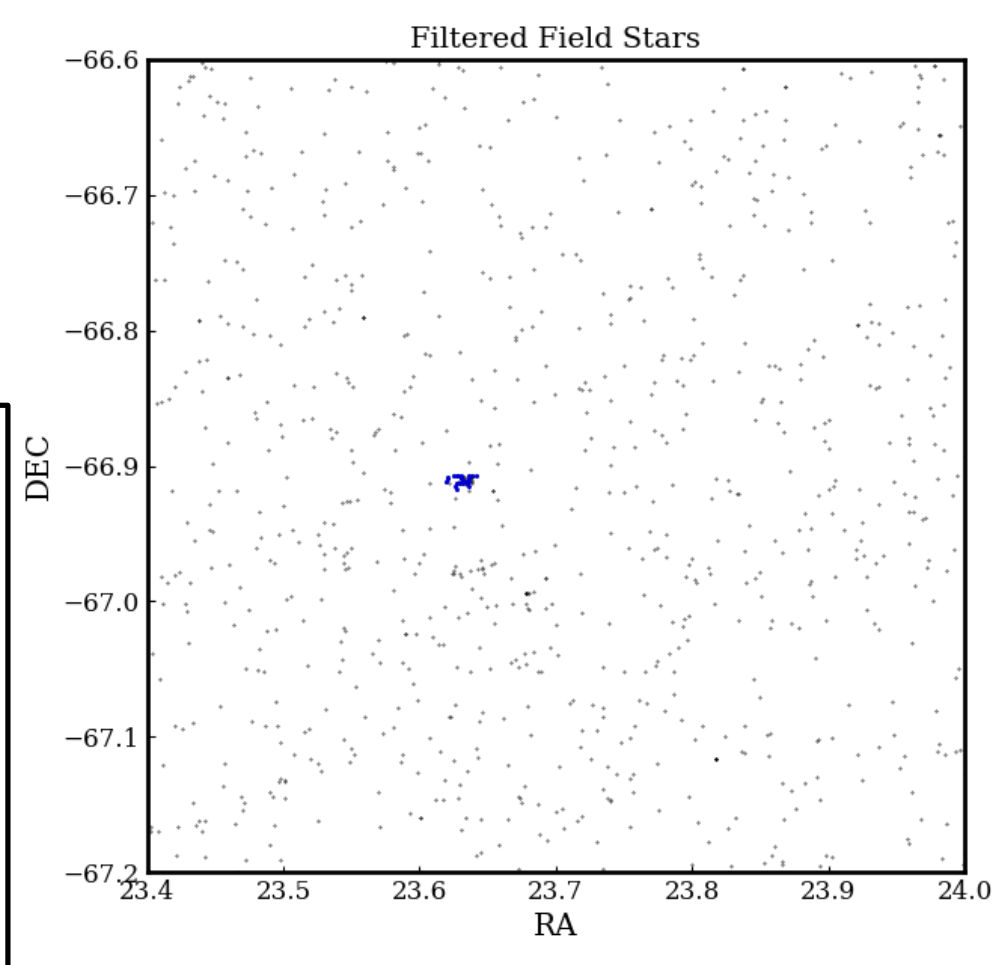




\section{Searching for Dwarf Galaxies: Example Result}
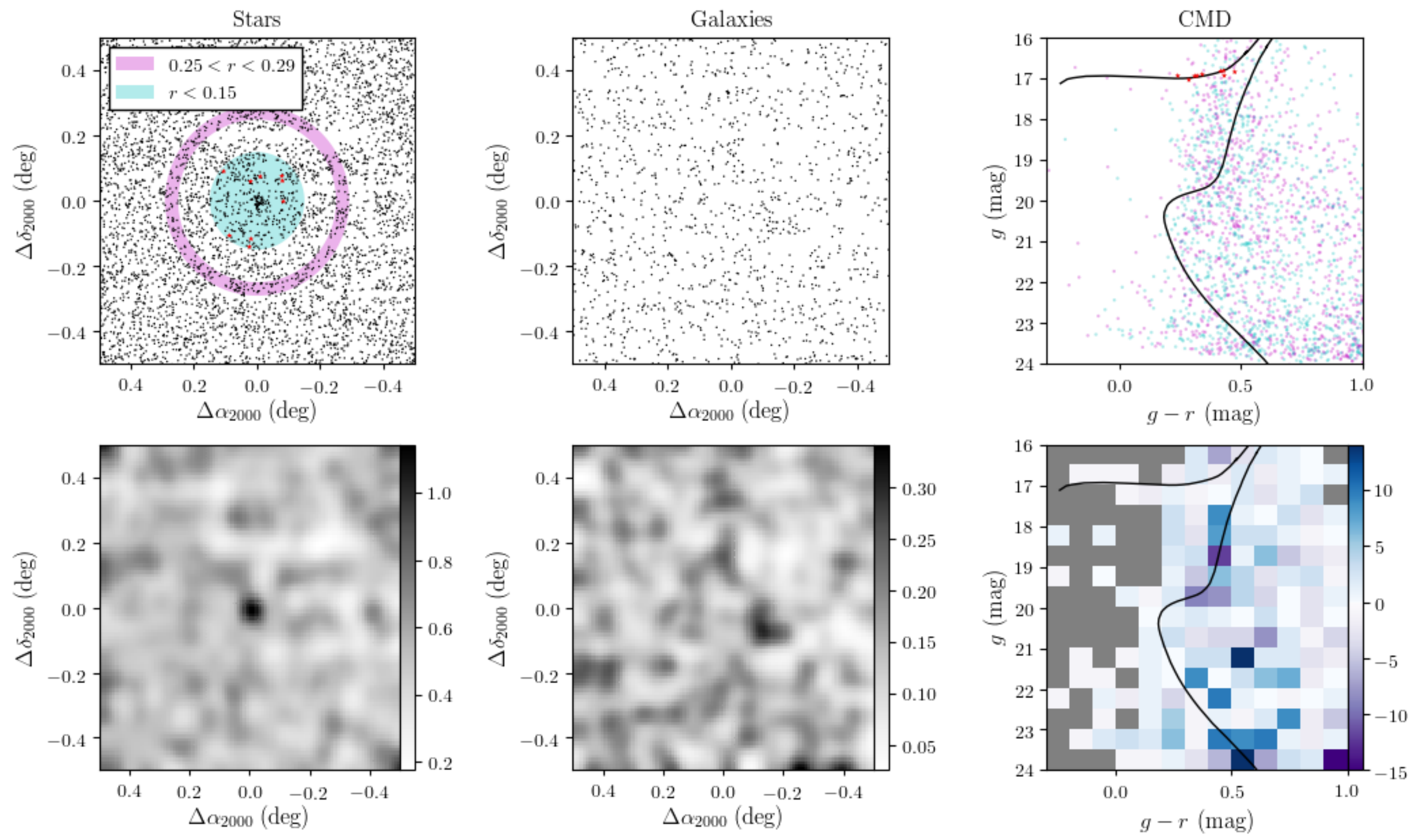


\section{Characterizing Candidates, continued}

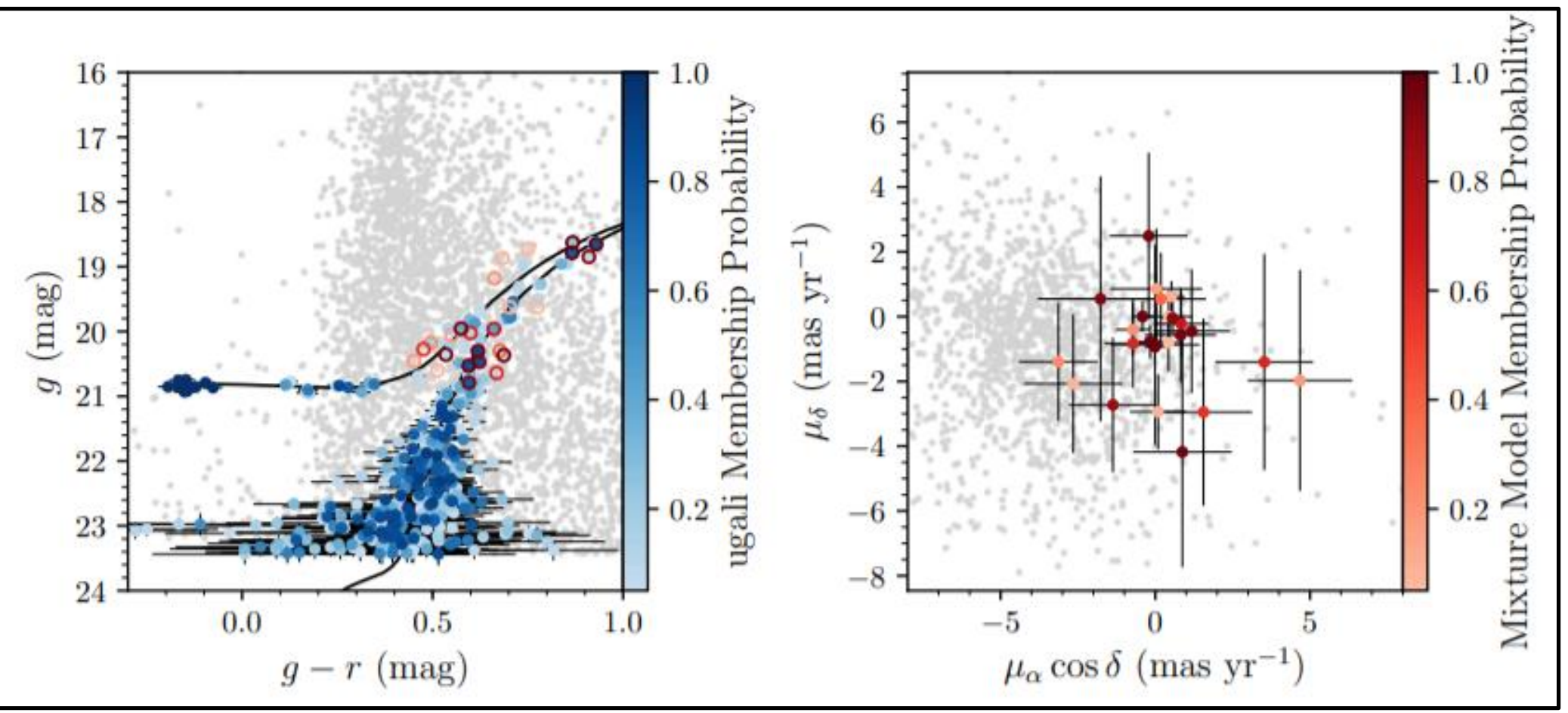

Centaurus 1, from Mau and Cerny et. al (2020)
Cross compare bright member stars to high precision proper motion measurements from the Gaia satellite.

Clustering of stars in the righthand plot suggests bulk motion of Gravitationally bound stars

$\rightarrow$ real system! 


\section{Classifying Candidates}

In general, halo star clusters tend to be:

- Higher (brighter) surface brightness

- More compact,

- More metal rich,

- Closer,

- Less elliptical,

- Older, and

- Fainter integrated brightness

compared to known dwarf galaxies.

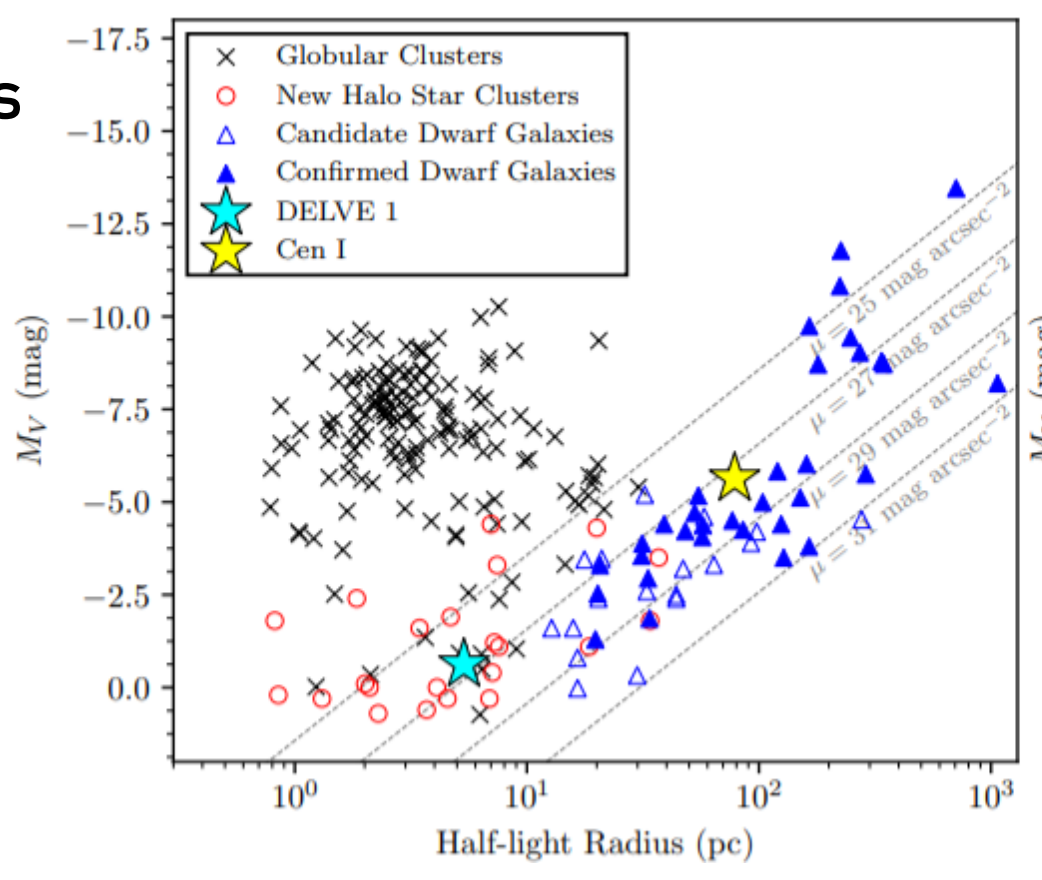

from Mau and Cerny et. al (2020)

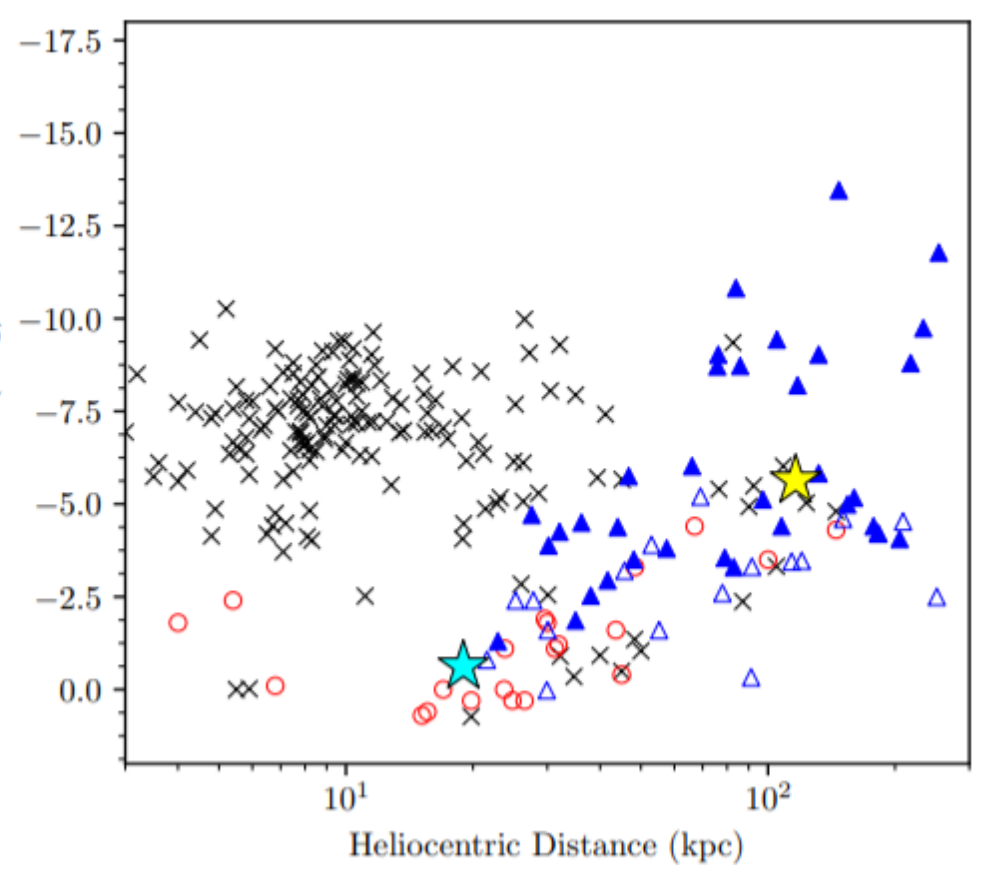

However: the classification of ultra-faint systems is not always clear! 


\section{Follow-Up Studies}

To maximize scientific insight from dwarf galaxy systems, deeper imaging and spectroscopy is required

Deeper + Time-Series Imaging:

- Deeper imaging allows for resolving fainter member stars, leading to better structural parameters

- Time-Series imaging allows for searches for variable stars, especially RR Lyrae $\rightarrow$ precise distances

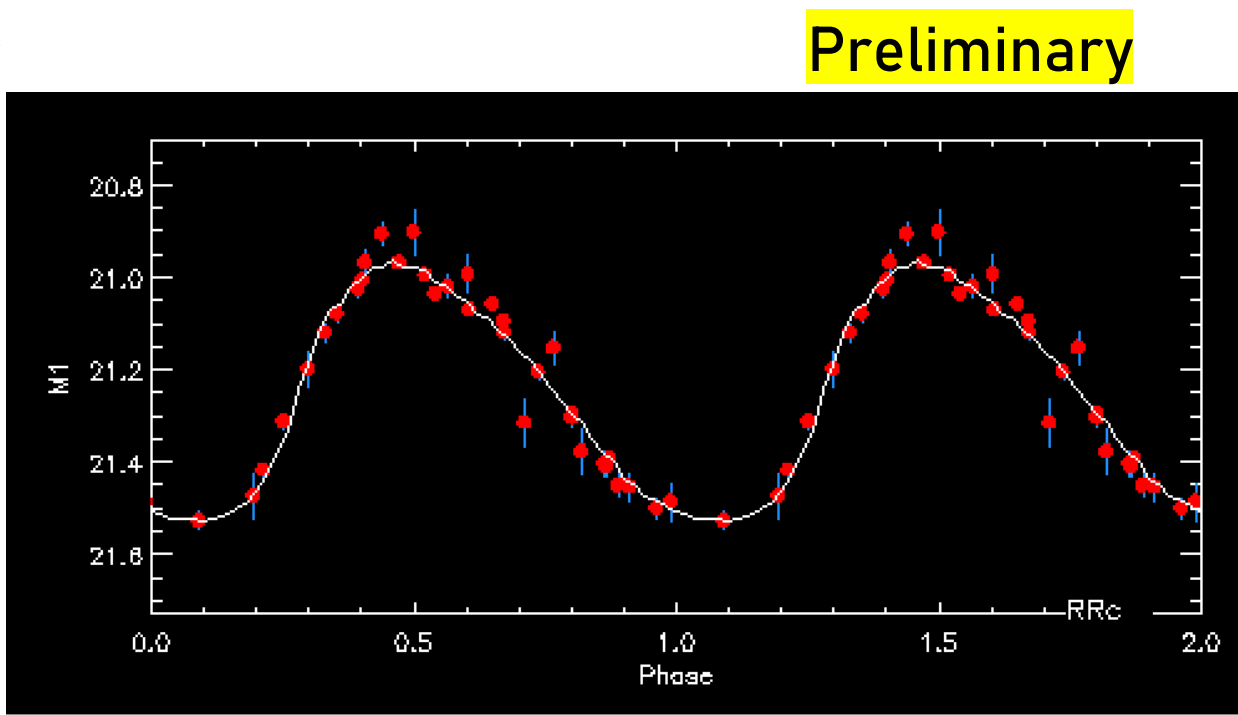

Spectroscopy:

- Understanding the elemental abundances within the system can help demystify its origin and history

- Ex: Large spread of metallicities suggests multiple stellar populations/periods of star formation

Candidate RR Lyrae variable star in Cen I: produces distance consistent with initial discovery results

- Resolving stellar velocities can allow for estimates of system dynamical mass -> insight into dark matter content 


\section{Future Work}

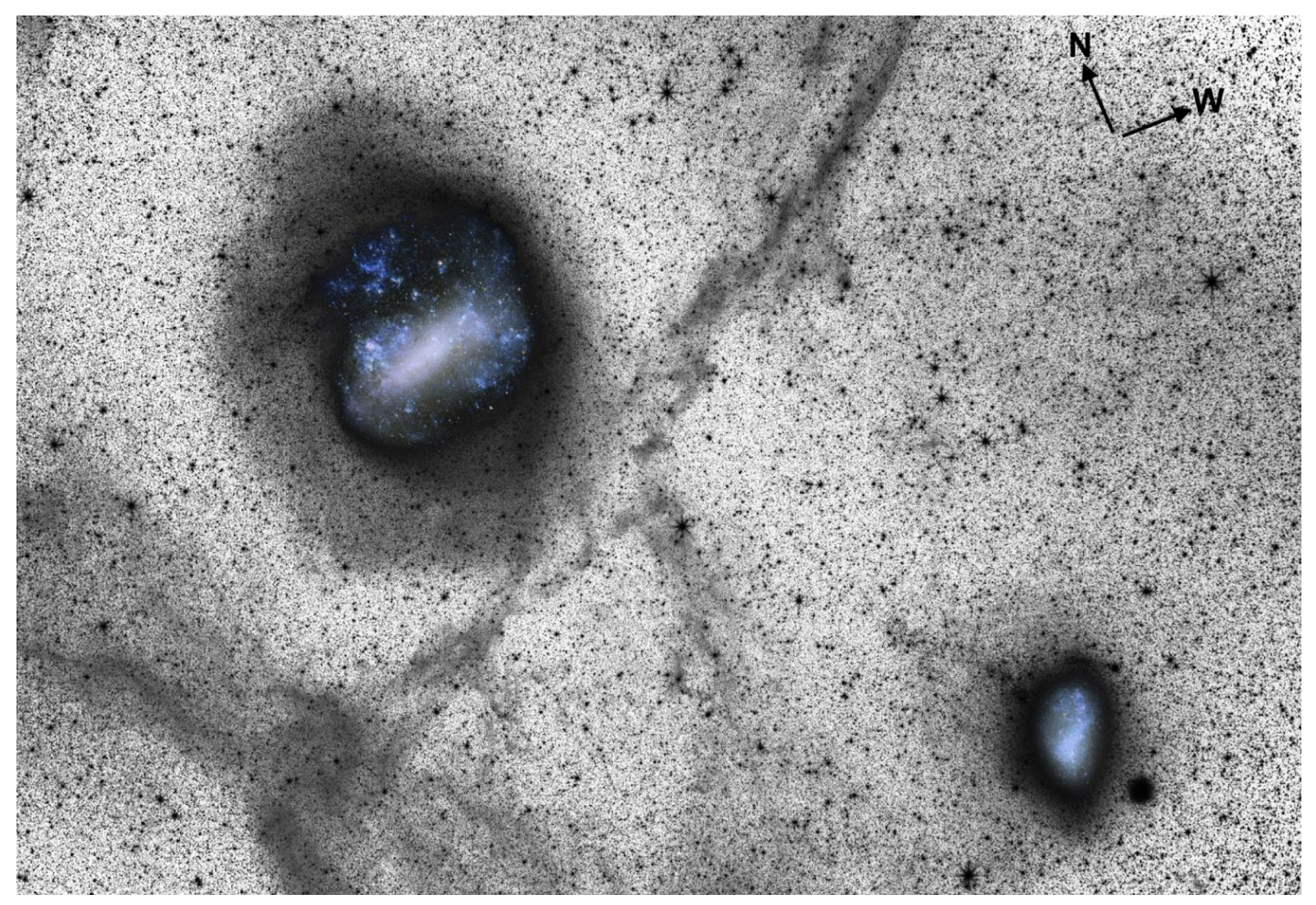

Image Credit: Besla et al (2016).
- Search over different regions of the sky especially around the Large and Small Magellanic Clouds

- "Hierarchical" galaxy formation suggests that even satellite galaxies can host satellite galaxies

- Growing evidence that the LMC and SMC have "brought in" their own satellites as they infall into the Milky Way 